\title{
Doped Silicon Nanowires for Lithium Ion Battery Anodes
}

\author{
Omer Salihoglu ${ }^{a} \oplus^{\circledR}$, Yasser El Kahlout ${ }^{a}$ \\ ${ }^{a}$ TÜBİTAK Marmara Araștırma Merkezi, 46470, Kocaeli, Turkey
}

Received: April 25, 2018; Revised: September 17, 2018; Accepted: December 04, 2018

\begin{abstract}
Nanostructured silicon ( $\mathrm{Si}$ ) has showed outstanding results as Li-ion battery anode material. Fabrication of nanostructured silicon anode materials is usually very complex, time consuming and expensive. In this work, silicon nanowires (SiNW`s) were produced by using rapid and uncostly metal catalyzed electroless etching (MCEE) method from various silicon wafers with different dopant atoms and concentrations. We have investigated the effect of doping level on capacities and cycle stability. Highly doped silicon nanowires produced better results than lightly doped silicon nanowires due to their highly conductive and highly porous nature. Arsenic doped silicon nanowire anode electrodes have reached a capacity of $3635 \mathrm{mAh} / \mathrm{g}$ for the first lithiation and maximum $25 \%$ charge capacity loss after the $15^{\text {th }}$ cycle. Owing to their small size and porosity this highly doped silicon nanowires showed very high performance and cycle retention as a lithium ion battery anode material.
\end{abstract}

Keywords: silicon nanowire, SiNW, anode material, lithium ion, Li-Ion, MCEE.

\section{Introduction}

Lithium ion has become a main rechargeable battery technology for most applications due to its superior performance as compared to other battery chemistries. Despite this fact, an improvement still has to be made to increase capacity and reduce size and weight of the Li-Ion batteries for the high power density demanding applications such as electric vehicles ${ }^{1}$. Silicon based anode electrodes get immense attention by the battery researchers around the globe ${ }^{2-4}$ for its encouraging charge capacity of $(4000 \mathrm{mAh} / \mathrm{g}$ which is almost 10 times higher than commercial anode materials ${ }^{5}$. Beside this, $\mathrm{Si}$ is environmentally friendly and it is the second most abundant material in the earth crust. Finally, silicon anode has relatively low discharge potential $((0.5 \mathrm{~V}$ vs. $\left.\mathrm{Li} / \mathrm{Li}^{+}\right)$which guarantees that high operation voltages can still be reached by using it against classical cathodes. However, silicon swells up to $320 \%$ by volume on lithium insertion (lithiation), and can contract dramatically on lithium extraction (delithiation). This can result in pulverization, loss of electrical contact and an unstable solid electrolyte interface (SEI) which causes rapid capacity losses. To overcome these problems, nanostructured silicon materials including nanowires, nanopowders, nanotubes, nanoporous films, nanocrystals, and core-shell nano fibers have been proposed in literature. The strain in such silicon nanostructures can be relaxed without mechanical degradation due to their high surface to volume ratio ${ }^{6}$. Beside their resistance to pulverization, silicon nanotubes reduce the electrical resistance by creating good conductive network channels. Therefore silicon nanowires show excellent performance and electrochemical stability when used as anodes for rechargeable Li-ion batteries.

*e-mail: omersalihoglu@yahoo.com.
Many different techniques have been used to fabricate Silicon nanowire anodes, including thin film deposition ${ }^{7}$, interference lithography ${ }^{8}$, nanoimprint lithography (NIL) ${ }^{5}$, deep reactive ion etching ${ }^{9}$, and vapor-liquid-solid (VLS) ${ }^{10}$.

Most of these production methods generally involve expensive and/or complex processes such as high temperatures, plasma deposition and etching with expensive templates. Metal assisted chemical etching is a simple and low cost method to fabricate Silicon nanoporous wires. In this process, Si wafer is etched by anodic etching in hydrofluoric (HF)/ $/ \mathrm{ANO}_{3}$ containing aqueous solutions. MCEE enables advanced silicon wire configuration where roughness and porosity within the nanowire can be controlled. MCEE solution contains metal ions and HF solutions. Ag particles on the silicon substrate catalyze HF etch, resulting in silicon nanowires or pores on the silicon surface. Huang et al. ${ }^{11}$ used $90 \mu$ m thick silicon wafer to fabricate total $90 \mu \mathrm{m}$ long silicon nanowires. After the $10^{\text {th }}$ cycle, capacity dropped from $2000 \mathrm{mAhg}^{-1}$ to 1000 $\mathrm{mAhg}^{-1}$. However, it showed a promising cycling ability after 30 cycles with capacity around $900 \mathrm{mAhg}^{-1}$. Furthermore, $\mathrm{M}$. Ge et al. ${ }^{12}$ reported using porous silicon nanowire anode materials. When used with alginate binder, nano porous $\mathrm{Si}$ anode showed $2000 \mathrm{mAh} / \mathrm{g}$ capacity even after 250 cycles.

In this work, we demonstrate the use of porous silicon nanowire films as an anode active material for lithium ion batteries. Silicon nanowires have been fabricated by using metal catalyzed electroless etching (MCEE) method on lightly and highly doped $\mathrm{Si}(100)$ wafers. MCEE is a simple, inexpensive and efficient method to produce the mass production of silicon nanowire battery anodes. The process only uses aqueous solution of silver nitrate (AgNO3) and hydrofluoric acid (HF) in room temperature enhancing its superiority over all other methods (Plasma enhanced chemical vapor deposition, reactive ion etching, chemical vapor deposition, 
vapor liquid solid growth ...). After nanowire growth on $\mathrm{Si}$ wafer, nanowires were raptured from the Si wafer by short sonication to get dispersed nanoparticles inside ethyl alcohol solution. Performance of the cells compared against lithium metal cathode in button cell battery configuration. Three different wafers have been used; $p$ type lightly boron doped, $\mathrm{p}$ type highly boron doped and $\mathrm{n}$ type highly arsenic doped wafers with resistivities of $30 \Omega \mathrm{cm}, 0.005 \Omega \mathrm{cm}$ and 0.005 $\Omega \mathrm{cm}$, respectively. These silicon wafers were obtained doped with the above specified characteristics from UniversityWafer, Inc. Effects of the doping concentration and dopant type on battery capacity and cycling ability were discussed.

\section{Experimental}

The production of silicon nanowire by using MCEE method is quite simple and quick technique to create perfectly aligned one dimensional (1D) nanoporous silicon arrays. Single step MCEE method was adapted from K. Peng et al. ${ }^{13}$. The chemicals that are used in this work were ordered from Sigma Aldrich and used without further purification. Prior to process, the silicon wafers were cleaned in warm acetone, then methanol, and then warm isopropanol in an ultrasonic bath for 5 minutes each. To remove organic residues from the silicon samples, wafers were dipped into piranha solution for 30 minutes $\left(\mathrm{H}_{2} \mathrm{O}_{2} / \mathrm{H}_{2} \mathrm{SO}_{4}\right.$ by volume ratio of $\left.1 / 3\right)$. To remove native oxide layer from the surface, wafers were immersed into diluted HF solution (1/30 by volume) for 30 minutes. The MCEE solution was prepared by combining $0.02 \mathrm{M}$ $\mathrm{AgNO}_{3}$ and 4.6 M HF solutions in a HF resistant container. The silicon samples were then dipped into MCEE solution at room temperature for two hours to form about $20 \mu \mathrm{m}$ long silicon nanowires. Chemical reaction terminated by immersing wafers into DI water. To remove silver dendrites from the surface of the silicon wafers, samples were dipped into diluted nitric acid solution $\left(1 / 3: \mathrm{HNO}_{3} / \mathrm{H}_{2} \mathrm{O}\right)$ for 30 minutes. Finally, samples were dipped into diluted HF solutions to remove native oxide layers and were rinsed in deionized water. Etch rate was determined as $(10 \mu \mathrm{m} /$ hour (Figure 1.a). Dispersed nanowires inside ethanol were achieved by sonication inside ethanol for about 5 seconds. Figure 1.c depicts images before and after sonication. Nanowires/ethanol solution has been drop casted on silicon wafer to see individual nanowires on the flat surface (Figure 1.b). The diameters of silicon nanowires vary between 80 and $120 \mathrm{~nm}$.

Formation of 1D silicon nanowires can be explained on the basis of a self-assembled microscopic electrochemical cell theory ${ }^{14}$. Soaking Si wafer into the solution reduces Ag ions to form nanoparticles which are uniformly distributed on the surface. Silicon underneath of the Ag particle gets anodically oxidized due to electron transfer between $\mathrm{Ag}$ and Si. Anodically oxidized silicon is etched away by HF. These reactions are spontaneous and continue until Si wafer is removed from the solution.
The silicon nanowire solution (dispersed in ethanol), carbon black, and polyvinylidene fluoride (PVDF) were mixed together with n-methyl-2-pyrrolidone (NMP) to form the slurry to be used in fabricating the anode. The weight ratio of silicon nanowire to PVDF to carbon black was kept constant at 80:16:4. We have prepared large batch solution of the PVDF and carbon black in low concentration then we have used micro pipet to weigh them precisely. Then, the slurry was drop casted onto copper foil (Circle pieces with a radius of $13 \mathrm{~mm}$ ) and was placed into an oven at 125 $0 \mathrm{C}$ to remove all solvents from the coating. Active material loadings have been varied between $0.25 \mathrm{mg}$ and $0.30 \mathrm{mg}$. As a counter electrode a thin film of lithium metal was used. Lithium hexafluorophosphate (LiPF6) was used as an electrolyte and Whatman microfiber (GF/D) was used as a separator. Finally, the components were assembled into a coin cell (Figure 1.d) inside an argon filled glove box to evaluate the electrochemical performance of silicon nanowire anodes. All measurements were executed inside an argon filled glove box.

\section{Results and Discussion}

To study the electrochemical properties of the silicon nanowire electrodes BST8-MA model (MTI corporation) battery analyzer was used. The cyclic properties of the cells were evaluated between 3 and $0.1 \mathrm{~V}$ with various charge/ discharge rates ranging from 0.3 to $1.2 \mathrm{~A} / \mathrm{g}$. The capacity calculated based on Si mass only. To determine the capacity from the non-silicon based sources, same electrode preparation procedures, except addition of silicon nanowires, have been used and the capacity of non-Si sources is measured as (20 $\mathrm{mAh}$ for all samples. An experiment was conducted to check the functionality of the pure silicone nanofiber. It was shown that the coin cell failed to work. Pure silicon nanofiber has very insulating nature which makes it impractical to be used alone. We have added only $4 \%$ percent of carbon black to get enough conductivity to create operational electrode. Figure 2.a shows the results of cyclic behavior of the cells with the SiNW's from $\mathrm{p}$ type lightly boron doped (Sample \#1), p type highly boron doped (Sample \#2) and n type highly arsenic doped (Sample \#3) wafers with resistivities of $30 \Omega \mathrm{cm}, 0.005 \Omega \mathrm{cm}$ and $0.005 \Omega \mathrm{cm}$, respectively. The first lithiation capacities of the sample \#1, \#2 and \#3 were measured as $3680 \mathrm{mAh} / \mathrm{g}, 3645 \mathrm{mAh} / \mathrm{g}$ and $3635 \mathrm{mAh} / \mathrm{g}$, respectively, indicating that initial capacities of the samples were almost same. After first cycle the lithiation capacities were measured as $661 \mathrm{mAh} / \mathrm{g}, 1507 \mathrm{mAh} / \mathrm{g}$ and $1792 \mathrm{mAh} / \mathrm{g}$ for sample \#1, \#2 and \#3, which indicates $82 \%, 58 \%$ and $50 \%$ capacity losses at $2^{\text {nd }}$ cycle, respectively. We believe that these loses are due to the formation of electrically isolated active material particles during the lithium insertion and extraction. These irreversible losses are slightly high for the practical usage of SiNW electrodes in commercial batteries; 

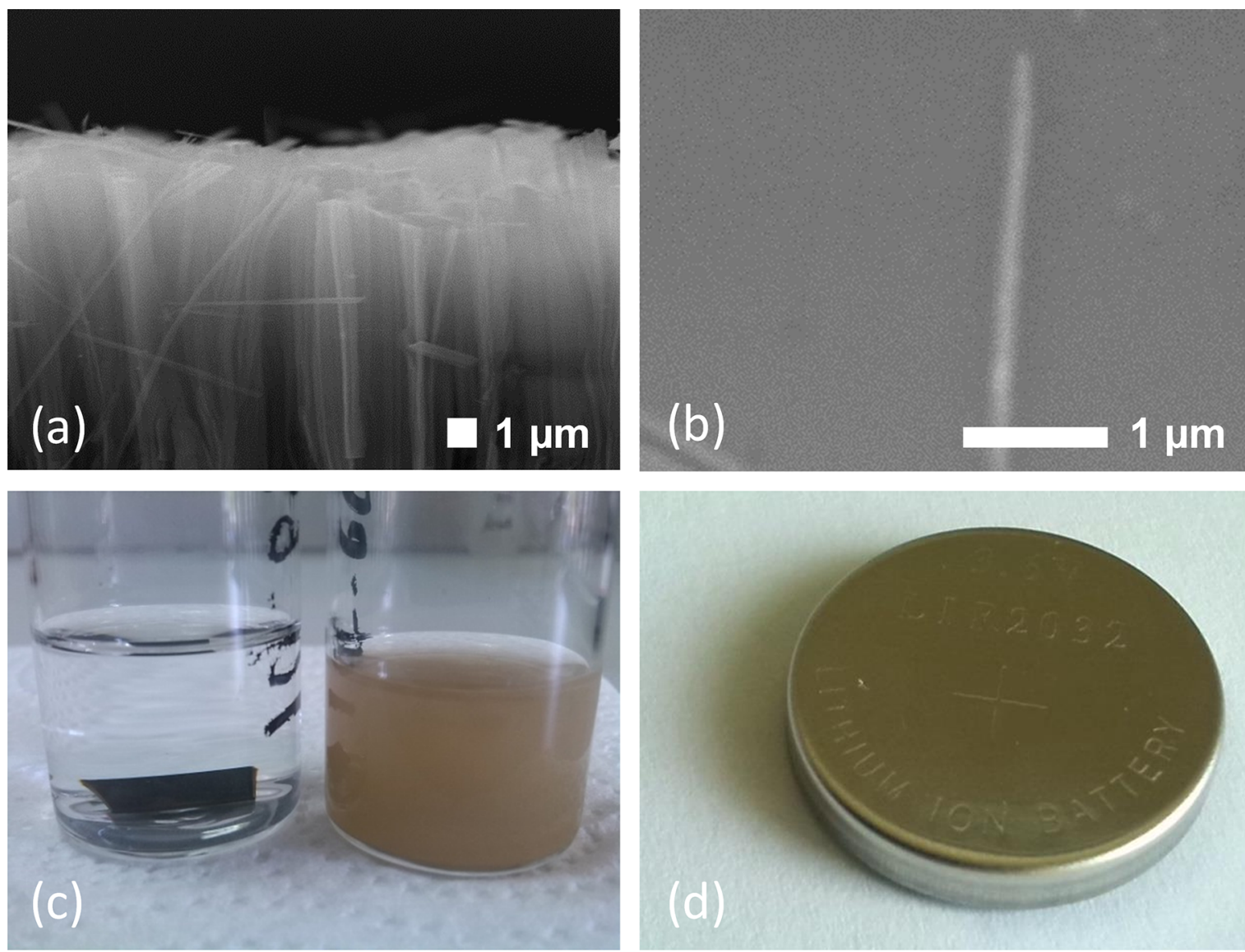

Figure 1. a) SEM cross section image of nanowires attached on silicon wafer. b) Individual silicon nanowires on flat silicon surface. c) Silicon nanowires before and after sonification. d) Assembled coin cell.

some methods should be used to fix this problem such as coating carbon or using a conductive polymer as a binder. The Coulombic efficiency of $99 \%$ was reached after the first several cycles which is required for commercialization of silicon based batteries ${ }^{15}$. Highly doped SiNW's show better cycle retention than lightly doped SiNW's. This might be due to better electrical conduction between isolated silicon crystals. As another explanation, highly doped silicon wafer may cause denser pore structure formation than lightly doped silicon wafer. Wang et al. showed that, the pore size is dramatically increased by the doping concentration ${ }^{16}$. Figure $2 \mathrm{~b}$ shows Galvanostatic $1^{\text {st }}$ and $16^{\text {th }}$ charge/discharge profiles of the silicon nanowire electrodes cycled between $0.1 \mathrm{~V}$ to 3.0 volt at $0.3 \mathrm{~A} / \mathrm{g}$ rate. Sample \#1 showed highest capacity at first delithiation but it degraded very quickly and gave lowest capacity at $16^{\text {th }}$ charge. Sample \#2 and sample \#3 showed similar capacities at first charge but sample \#3 showed average of $10 \%$ better capacity than sample \#2 at the $16^{\text {th }}$ charge profile.

This capacity loss is attributed to degradation of silicon structure by volume change during lithiation and delithiation processes ${ }^{17}$. Highly doped silicon nanowires withstand better than lightly doped silicon nanowires due to high electrical connection and high porous nature of MCEE fabricated highly doped silicon nanowires ${ }^{16,18}$. Excessive foreign atoms in highly doped silicon nanowires create disordered crystal structure to accommodate the volume change and provide stability against the lithiation/delithiation process ${ }^{19,20}$. Furthermore, arsenic doped sample (sample \#3) and boron doped sample (sample \#2) have shown similar capacities. Under our experimental conditions, doping concentration is much more dominant parameter for the battery performance. Our experimental results show that $\mathrm{n}$ type dopants show slightly higher capacity than $p$ type dopants. The uncertainties in the wafer doping concentrations in the manufacturing process can be the cause of this slight capacity difference. Therefore, it is difficult to generalize the conclusion of this experiment.

Cyclic voltammogram (CV) curve of the sample have been taken by using Methrom Autolab M101 Potentiostat/ Galvanostat module. ACV of the highly doped $\mathrm{n}$ type silicon nanowires film electrode from $0.01 \mathrm{~V}$ to $1.00 \mathrm{~V}$ at $0.1 \mathrm{mV} / \mathrm{s}$ rate is shown in Fig. 3. Continuous lithiation starts from $0.3 \mathrm{~V}$ and ends at cut off voltage $(0.01 \mathrm{~V})$. The peaks at $0.02 \mathrm{~V}$ and $0.19 \mathrm{~V}$ in the cathodic process correspond to the conversion of crystalline $\mathrm{Si}$ to the $\mathrm{Li}_{x} \mathrm{Si}$ phase, while the two peaks at $0.32 \mathrm{~V}$ and $0.49 \mathrm{~V}$ in anodic process correspond to the 

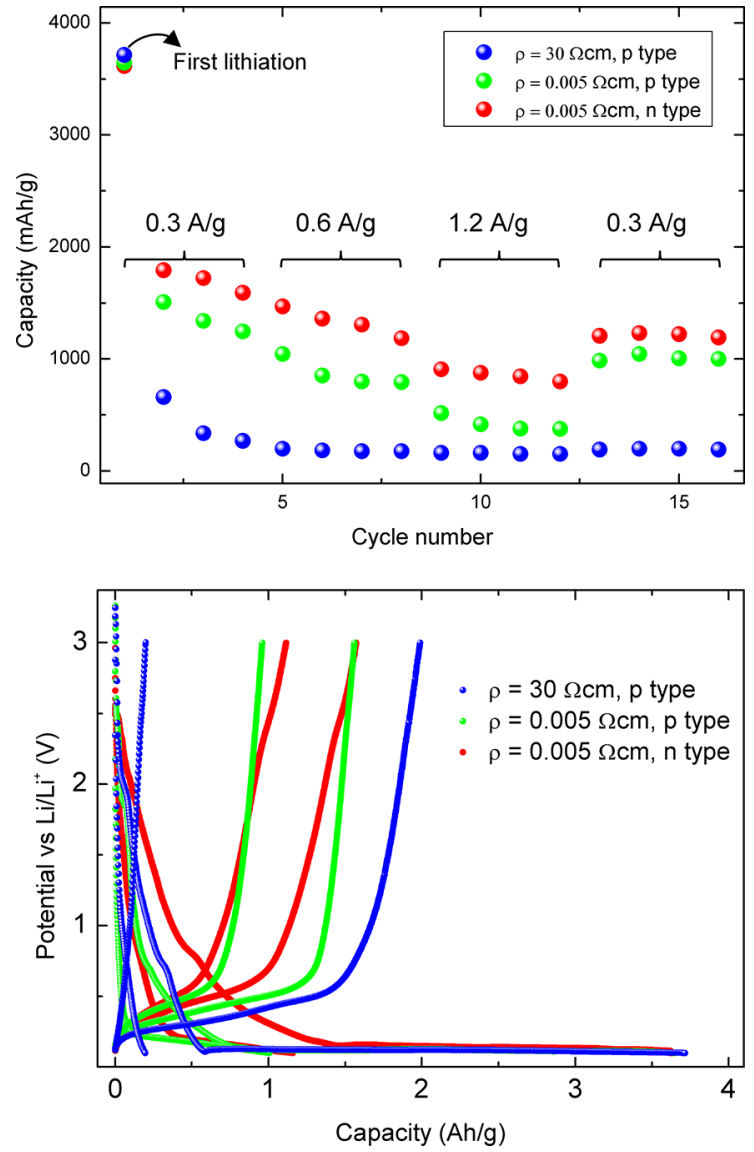

Figure 2. a) Cyclic behaviors of the cells with the nanowires from $\mathrm{p}$ type lightly boron doped, $\mathrm{p}$ type highly boron doped and $\mathrm{n}$ type highly arsenic doped wafers with resistivities of $30 \Omega \mathrm{cm}, 0.005$ $\Omega \mathrm{cm}$ and $0.005 \Omega \mathrm{cm}$, respectively. b) Galvanostatic $1 \mathrm{st}$ and $16 \mathrm{th}$ lithiation/delithiation profiles of the silicon nanowire electrodes cycled between $0.1 \mathrm{~V}$ to 3.0 volt at $0.3 \mathrm{~A} / \mathrm{g}$ rate.

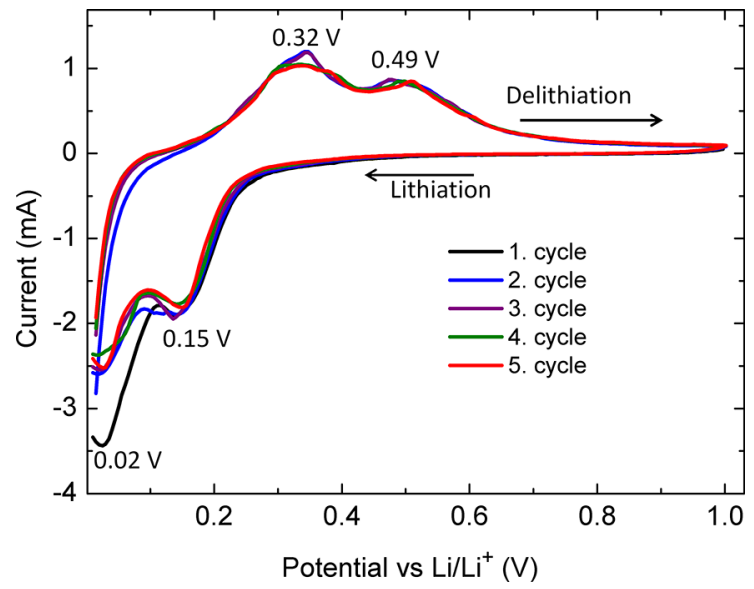

Figure 3. Cyclic voltammogram $(\mathrm{CV})$ versus $\mathrm{Li} / \mathrm{Li}+$ profiles of $\mathrm{n}$ type highly doped nanowire electrode (sample \#3) at a scan rate of $0.1 \mathrm{mV} / \mathrm{s}$ over the potential window of $0.01 \mathrm{~V}$ to $1.00 \mathrm{~V}$ for the first 5 cycles.

delithiation of amorphous (-Li $\mathrm{Si}$ to (-Si. These four lithiation and delithiation peaks are often reported in the literature for both silicon nanowires ${ }^{4}$ and silicon nanoparticles ${ }^{21}$.

Figure 4 shows the voltage profiles for $1^{\text {st }}$ to $3^{\text {rd }}$ and $14^{\text {th }}$ to $16^{\text {th }}$ galvanostatic delithiation/lithiation cycles of $n$ type highly doped nanowires (sample \#3). The capacities and capacity retentions of the samples were cycled at $0.3 \mathrm{~A} / \mathrm{g}$. The first discharge and charge capacities of the silicon nanowire film electrode were $3615 \mathrm{mAh} / \mathrm{g}$ and $1569 \mathrm{mAh} / \mathrm{g}$, respectively. The initial capacity losses mostly arise from formation of SEI on Si active material and irreversible trapping of inserted lithium ions by the Silicon nanowire network. The electrodes lost $25 \%$ of the initial charge capacity after $15^{\text {th }}$ cycle. The sample was not further purified to eliminate the large chunks of silicon particles. This might be a reason for the capacity loss that is observed during the first couple of cycles. Large particles have less structural stability against the volume change during lithiation/delithiation process ${ }^{22}$. The cycle retention is determined as $0.27 \%$ decay per cycle for silicon nanowire anodes after the $10^{\text {th }}$ cycle. These results were acquired by using PVDF binder which has been known as very inefficient binder for Si based anode electrodes ${ }^{23}$. Using alginate ${ }^{12}$, PEDOT (Poly(3,4-ethylenedioxythiophene) $)^{23}$ or PAA (poly(acrylic acid)) ${ }^{24}$ as a binder may improve cycling stability.

For a high energy battery system, the use of a high capacity anode material is a crucial factor. An ideal anode material should have high energy density and high cycle life. It should also remain structurally stable and electrically conductive after many charge/discharge cycles. Highly porous silicon nanowires may satisfy all these needs. Unlike lightly doped silicon nanowires, highly doped silicon wafer resulted in highly conductive and highly porous nanowires which lead to a better cycling stability. The high internal porosity facilitates the volume change of Si nanowires without pulverizing the solid electrolyte interphase (SEI) at the outer surface ${ }^{25}$. The strain in nanostructures can be relaxed easily, without mechanical fracture, because of their small size and available surrounding free space ${ }^{26}$. At the crystal surface,

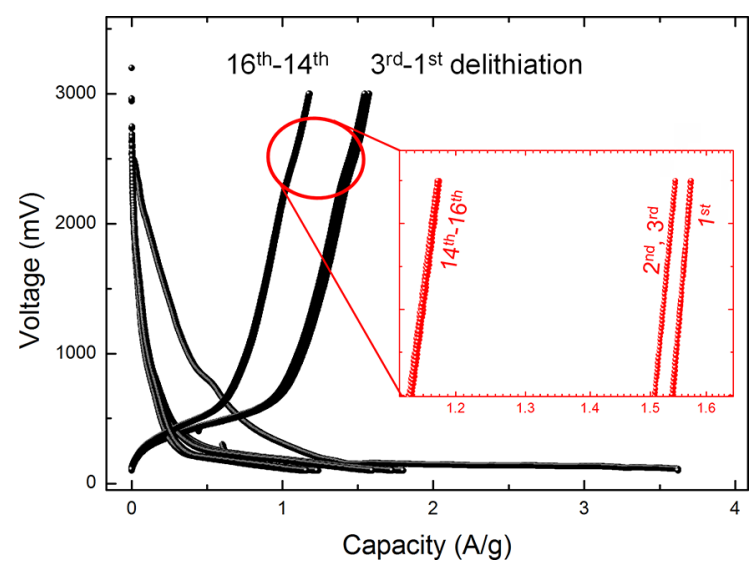

Figure 4. Galvanostatic delithiation/lithiation profiles of silicon nanowire electrode from $\mathrm{n}$ type $0.005 \Omega \mathrm{cm}$ wafer. Inset shows close up image of the red circled part. 
defects and impurities act as nucleation centers for pore formation ${ }^{27}$. As a result higher dopant concentrations create higher pore density and highly rough nanowire surfaces ${ }^{27}$. Although silicon nanowires are very promising, some very important challenges still need to be improved before commercialization of this technology. The capacity of the nanostructured Si electrodes can be seen to decay during charge/discharge cycle even for the highly doped silicon wafers. This decay is mostly attributed to deterioration of the Si structure, likely through loss of electrical contact to portions of the Si nanowire. The electrical contact loss is a result of the high volume change of the Si electrodes and can be proceed through either rupturing of the Si segments or detachment of the nanowires from the copper current collector ${ }^{28}$. In addition to that, Silicon surface is easily oxidized and a native oxide layer of several nanometers thick is quickly formed upon exposure to air ${ }^{29}$. This thin oxide layer may negatively effects cell performance by reducing lithiation efficiency of the underlying silicon core and by reducing electrical conductivity of the individual silicon wires.

\section{Conclusion}

In this study, silicon nanowires were used as an anode electrode for lithium ion batteries. Nanowires were fabricated by using MCEE method from various silicon wafers with different dopant atoms and concentrations (Boron doped; ( = 30 and $0.005 \Omega \mathrm{cm}$, Arsenic doped; ( $=0.005 \Omega \mathrm{cm})$. We have mainly investigated effect of the doping concentration on the capacities and cycle retention of the SiNW based Li-ion batteries. The first lithiation capacities of the samples were measured as $3680 \mathrm{mAh} / \mathrm{g}, 3645 \mathrm{mAh} / \mathrm{g}$ and $3635 \mathrm{mAh} / \mathrm{g}$, which are very close to theoretical specific capacity of the silicon $(\sim 4000 \mathrm{mAh} / \mathrm{g})$. The second lithiation capacities showed $82 \%, 58 \%$ and $50 \%$ capacity losses for the p type lightly, $\mathrm{p}$ type highly and $\mathrm{n}$ type highly doped samples, respectively. Highly doped silicon wafers create highly porous nanowires which provide a safe volume expansion without damaging SEI. Furthermore, the strain in nanowires can be easily relaxed without mechanical fracture. Highly arsenic doped sample and highly boron doped sample showed similar performances as an active anode material. Therefore dopant concentration is much more important parameter. The initial capacity of the highly arsenic doped sample is degraded by around $25 \%$ after the $15^{\text {th }}$ cycle. Existence of large chunks of silicon nanowires inside the suspended solution is presumed to be the reason for these losses. According to the cyclic voltammogram spectra, continuous lithiation starts from $0.3 \mathrm{~V}$ and ends at cut off voltage $(0.01 \mathrm{~V})$. The peaks at $0.02 \mathrm{~V}$ and $0.19 \mathrm{~V}$ in the cathodic process correspond to the conversion of crystalline Si to the amorphous $\mathrm{Li}_{\mathrm{x}} \mathrm{Si}$ phase, while the two peaks at $0.32 \mathrm{~V}$ and $0.49 \mathrm{~V}$ in anodic process correspond to the delithiation of amorphous $\mathrm{Li}_{\mathrm{x}} \mathrm{Si}$ to amorphous $\mathrm{Si}$. These four lithiation and delithiation peaks are often reported in the literature for silicon nanostructures. This work shows that highly doped silicon wafers produce higher performance and better cycle life for Li-ion batteries due to porous and electrically conductive nature of the resulted nanowires.

\section{REFERENCES}

1. Lu L, Han X, Li J, Hua J, Ouyang M. A review on the key issues for lithium-ion battery management in electric vehicles. Journal of Power Sources. 2013;226:272- 288.

2. Peng KQ, Wang X, Li L, Hu Y, Lee ST. Silicon nanowires for advanced energy conversion and storage. Nanotoday. 2013;8(1):75-97.

3. Chan CK, Peng H, Liu G, McIlwrath K, Zhang XF, Huggins RA, et al. High-performance lithium battery anodes using silicon nanowires. Nature Nanotechnology. 2008;3:31-35.

4. Zamfir MR, Nguyen HT, Moyen E, Lee YH, Pribat D. Silicon nanowires for Li-based battery anodes: a review. Journal of Materials Chemistry A. 2013;1(34):9566-9586.

5. Mills E, Cannarella J, Zang Q, Bhadra S, Arnold CB, Chou SY. Silicon nanopillar anodes for lithium-ion batteries using nanoimprint lithography with flexible molds. Journal of Vacuum Science \& Technology B. 2014;32(6):06FG10.

6. Deshpande R, Cheng YT, Verbrugge MW. Modeling diffusioninduced stress in nanowire electrode structures. Journal of Power Sources. 2010;195(15):5081-5088.

7. Ohara S, Suzuki J, Sekine K, Takamura T. A thin film silicon anode for Li-ion batteries having a very large specific capacity and long cycle life. Journal of Power Sources. 2004;136(2):303306.

8. Choi WK, Liew TH, Dawood MK, Smith HI, Thompson CV, Hong MH. Synthesis of Silicon Nanowires and Nanofin Arrays Using Interference Lithography and Catalytic Etching. NanoLetters. 2008;8(11):3799-3802.

9. Li J, Yue C, Yu Y, Chui YS, Yin J, Wu Z, et al. Si/Ge core-shell nanoarrays as the anode material for 3D lithium ion batteries. Journal of Materials Chemistry A. 2013;1(45):14344-14349.

10. Cui LF, Ruffo R, Chan CK, Peng H, Cui Y. Crystalline-Amorphous Core-shell Silicon Nanowires for High Capacity and High Current Battery Electrodes. NanoLetters. 2008;9(1):491-495.

11. Huang R, Zhu J. Silicon nanowire array films as advanced anode materials for lithium-ion batteries. Materials Chemistry and Physics. 2010;121(3):519-522.

12. Ge M, Rong J, Fang X, Zhou C. Porous Doped Silicon Nanowires for Lithium Ion Battery Anode with Long Cycle Life. NanoLetters. 2012;12(5):2318-2323.

13. Peng K, Wu Y, Fang H, Zhong X, Xu Y, Zhu J. Uniform, AxialOrientation Alignment of One-Dimensional Single-Crystal Silicon Nanostructure Arrays. Angewandte Chemie International Edition. 2005;44(18):2737-2742.

14. Qui T, Wu XL, Yang X, Huang GS, Zhang ZY. Self-assembled growth and optical emission of silver-capped silicon nanowires. Applied Physics Letters. 2004;84(19):3867-3869. 
15. Wu H, Yu G, Pan L, Liu N, McDowell MT, Bao Z, et al. Stable Li-ion battery anodes by in-situ polymerization of conducting hydrogel to conformally coat silicon nanoparticles. Nature Communications. 2013;4:1943.

16. Wang D, Ji R, Du S, Albrecht A, Schaaf P. Ordered arrays of nanoporous silicon nanopillars and silicon nanopillars with nanoporous shells. Nanoscale Research Letters. 2013;8:42.

17. Xiao J, Xu W, Wang D, Choi D, Wang W, Li X, et al. Stabilization of Silicon Anode for Li-Ion Batteries. Journal of The Electrochemical Society. 2010;157(10):A1047-A1051.

18. Li S, Ma W, Zhou Y, Chen X, Xiao Y, Ma M, et al. Fabrication of porous silicon nanowires by MACE method in $\mathrm{HF} / \mathrm{H}_{2} \mathrm{O}_{2} /$ $\mathrm{AgNO}_{3}$ system at room temperature. Nanoscale Research Letters. 2014;9(1):196.

19. Hochbaum AI, Gargas D, Hwang YJ, Yang P. Single Crystalline Mesoporous Silicon Nanowires. NanoLetters. 2009;9(10):35503554 .

20. McSweeney W, Geaney H, O'Dwyer C. Metal-assisted chemical etching of silicon and the behavior of nanoscale silicon materials as Li-ion battery anodes. Nano Research. 2014;8(15):1395-1442.

21. Magasinski A, Dixon P, Hertzberg B, Kvit A, Ayala J, Yushin G. High-performance lithium-ion anodes using a hierarchical bottom-up approach. Nature Materials. 2010;9:353-358.

22. Kim H, Lee EJ, Sun YK. Recent advances in the Si-based nanocomposite materials as high capacity anode materials for lithium ion batteries. Materialstoday. 2014;17(6):285-297.
23. Yao Y, Liu N, McDowell MT, Pasta M, Cui Y. Improving the cycling stability of silicon nanowire anodes with conducting polymer coatings. Energy \& Environmental Science. 2012;5(7):7927-7930.

24. Magasinski A, Zdyrko B, Kovalenko I, Hertzberg B, Burtovyy $\mathrm{R}$, Huebner CF, et al. Toward Efficient Binders for Li-Ion Battery Si-Based Anodes: Polyacrylic Acid. ACS Applied Materials \& Interfaces. 2010;2(11):3004-3010.

25. Huang L, Wei Q, Sun R, Mai L. Nanowire electrodes for advanced lithium batteries. Frontiers in Energy Research. 2014;2:43.

26. Wu H, Chan G, Choi JW, Ryu I, Yao Y, McDowell MT, et al. Stable cycling of double-walled silicon nanotube battery anodes through solid-electrolyte interphase control. Nature Nanotechnology. 2012;7:310-315.

27. Qu Y, Zhou H, Duan X. Porous silicon nanowires. Nanoscale. 2011;3(10):4060-4068.

28. Zhao C, Li S, Luo X, Li B, Pan W, Wu H. Integration of Si in a metal foam current collector for stable electrochemical cycling in Li-ion batteries. Journal of Materials Chemistry A. 2015;3(18):10114-10118.

29. Li F, Balazs MK, Anderson S. Effects of Ambient and Dissolved Oxygen Concentration in Ultrapure Water on Initial Growth of Native Oxide on a Silicon (100) Surface. Journal of the Electrochemical Society. 2005;152(8):G669-G673. 